\title{
Multi-class dynamic network traffic flow propagation model with physical queues
}

C The Author(s) 2017. Published by Higher Education Press. This is an open access article under the CC BY license (http:// creativecommons.org/licenses/by/4.0)

\begin{abstract}
This paper proposes an improved multi-class dynamic network traffic flow propagation model with a consideration of physical queues. Each link is divided into two areas: Free flow area and queue area. The vehicles of the same class are assumed to satisfy the first-in-first-out (FIFO) principle on the whole link, and the vehicles of the different classes also follow FIFO in the queue area but not in the free flow area. To characterize this phenomenon by numerical methods, the improved model is directly formulated in discrete time space. Numerical examples are developed to illustrate the unrealistic flows of the existing model and the performance of the improved model. This analysis can more realistically capture the traffic flow propagation, such as interactions between multi-class traffic flows, and the dynamic traffic interactions across multiple links.
\end{abstract}

Keywords first-in-first-out (FIFO), multi-class traffic, physical queues, traffic flow modeling

\section{Introduction}

Modeling dynamic traffic flow propagation in a network is one of the key components of dynamic traffic assignment (DTA), which is one theoretical core in intelligent transportation system (ITS). The traffic flow model describes how traffic propagates inside a traffic network and hence governs the network performance in terms of travel time. The procedure in implementation is often called dynamic network loading (DNL). The performance of DTA models in terms of capturing actual travel behavior and computation speed relies on its traffic flow component.

Received June 7, 2017; accepted August 18, 2017

Yanfeng LI ( ( ), Jun LI

School of Economics and Management, Southwest Jiaotong University, Chengdu 610031, China

E-mail: yanwaa@126.com
Therefore, developing a realistic and efficient network traffic flow model is a basic and important work for the solution of DTA models.

Traditional DTA problems assume that homogeneous vehicles exist in the traffic network, and the related traffic flow propagation models govern only a single class of vehicles. However, various types of vehicles travel with different speeds and interact mutually in actual urban traffic network. The network traffic flow propagation model is required to capture the dynamics of heterogeneous mobility, such as the interactions between the multiclass vehicles and interactions across multiple links.

Existing traffic flow propagation models can be classified into two categories: Non-physical models and physical models. The former models have a simpler calculation but fail to capture some fundamental traffic dynamics such as queue spillback. The second category of models is also referred to advanced exit flow functions, which are developed based on either Daganzo's (1994; 1995) solution scheme (i.e., cell transmission model, CTM) or Newell's (1993) solution scheme to the Lighthill and Whitham (1955) and Richards (1956) (LWR) hydrodynamic model of traffic flow (Kuwahara and Akamatsu, 2001; Lo and Szeto, 2002a). These models can describe dynamic traffic conditions on a road network, including shock waves and the propagation of queues over links, and the related models are popularly applied to calculate travel times for DTA models in the past decade (Kuwahara and Akamatsu, 2001; Lo and Szeto, 2002b; Lo and Szeto, 2004; Szeto et al., 2011). Bliemer (2007) noted that those models rely on first-in-first-out (FIFO) rule, and cannot be extended to easily simulate multi-class traffic flow propagation in the network. Recently, Tuerprasert and Aswakul (2010) extended the single-class CTM to the multiclass CTM (MCTM) with heterogeneous vehicles. However, FIFO rule among each type of vehicles can be violated in the MCTM. This problem is the main difficulty in developing multi-class traffic flow models based on the LWR theory. 
In literature, the multi-class traffic flow models (Ran and Boyce, 1996; Lo et al., 1996; Huang and Lam, 2003; Logghe and Immers, 2008; Leclercq and Laval, 2007; van Wageningen-Kessels et al., 2010; Wong and Wong, 2002; Liu et al., 2013; Liu et al., 2017; Levin and Boyles, 2016) have received more and more attention due to their potential in representing traffic flow in a realistic manner. van Wageningen-Kessels (2016) developed a framework for the mathematically rigorous qualitative assessment of deterministic multi-class kinematic wave traffic flow models. Existing multi-class traffic flow propagation models can also be classified into two categories: Nonphysical models (Ran and Boyce, 1996; Lo et al., 1996; Huang and Lam, 2003; Logghe and Immers, 2008; Leclercq and Laval, 2007) and physical models (van Wageningen-Kessels et al., 2010; Wong and Wong, 2002; Liu et al., 2013; Liu et al., 2017; Levin and Boyles, 2016). Ran and Boyce (1996), Lo et al. (1996), Huang and Lam (2003) proposed the dynamic user optimal models with multiple users. However, these studies do not provide much attention to the actual multi-class traffic flow propagation. Logghe and Immers (2008) presented a multi-class traffic flow model by considering the road to be divided into parallel spaces used by each class separately. The interaction between different vehicle types in the same lane cannot be incorporated into the model. Leclercq and Laval (2007) developed a multi-class model with Lagrangian coordinates using a variational formulation, while van Wageningen-Kessels et al. (2010) proposed a Langrangian formulation of the kinematic wave model for multiple user classes. Wong and Wong (2002) extended the macroscopic LWR model to capture the behaviors with heterogeneous drivers. More recently, Liu et al. (2013) and Liu et al. (2017) developed a macroscopic emission model that considers the multi-class nature of traffic, and uses the models for model predictive control (MPC) for traffic networks. These models are only applied to a simple highway road without intersections. Levin and Boyles (2016) developed a multiclass cell transmission model with mixed autonomous and human traffic based on a collision avoidant car following model, and analysis indicated that as autonomous vehicles penetration rate increases, the capacity of a roadway increases. The nonphysical models have a simpler calculation but fail to capture some fundamental traffic dynamics, while physical models can capture the realistic traffic dynamics but are computationally inefficient. Therefore, it is necessary to develop multiclass dynamic traffic flow propagation models that can capture the realistic traffic dynamics and are computational efficient.

Bliemer (2007) developed an analytical multi-class DNL model that can capture the queue spillback and the interaction across various links. Since each whole link is divided into two parts (i.e., treated as two cells), its computational efficiency is considerably higher than that of classic numerical solution schemes for the multiclass
LWR model. However, we observed that Bliemer (2007)'s model can yield unrealistic inflow when the queue length approximates the length of the link and unrealistic outflow when the queue length decreases to zero. To cope with these unrealistic results, this paper improves Bliemer (2007)'s model by enhancing the sending and receiving functions of the model. In addition, Bliemer (2007)'s model is formulated in continuous space and cannot be solved analytically due to its complexity. To solve it by numerical methods, the improved model is directly formulated in discrete time space. We also develop numerical examples to illustrate the unrealistic flows generated by Bliemer (2007)'s model and the performance of the improved model.

The rest of this paper is organized as follows: The flow propagation model will be formulated in the next section, where a link travel time estimation method will also be presented. In Section 3, numerical examples are developed to illustrate the properties of the proposed model. Finally, Section 4 presents this study's conclusions.

\section{Model}

The multi-class traffic flow propagation model consists of a link model and a node model. The link model describes the dynamic propagation of various types of vehicles in links, while the node model presents the transmission from upstream links to downstream links.

\subsection{Notations}

Consider a traffic network $G=(N, A)$ with multiple origins and destinations, where $N$ is the set of nodes, and $A$ is the set of links. The time interval $[0, T]$ is discretized into $K$ time intervals. The length of each interval is $\delta$, which satisfies $K \delta=T$. As shown in Fig. 1, Link $a$ is divided into two areas: Free flow area and queue area. The length of the two areas are time varying, and denoted by $L_{a}^{\mathrm{f}}(k)$ and $L_{a}^{\mathrm{q}}(k)$ for a specified interval $k$ respectively. We have $L_{a}=L_{a}^{\mathrm{f}}(k)+L_{a}^{\mathrm{q}}(k)$, where $L_{a}$ is the length of Link $a$. The traffic capacities are identical along the whole link. There are $M$ types of vehicles with different free flow speeds in the network. All vehicles travel with their own free flow speed in the free flow area but with the same speed in the queue area. Therefore, FIFO rule is satisfied only for the vehicles of the same type. Different types of vehicles can overtake each other in the free flow area.

Using the cumulative flows, we can obtain the number

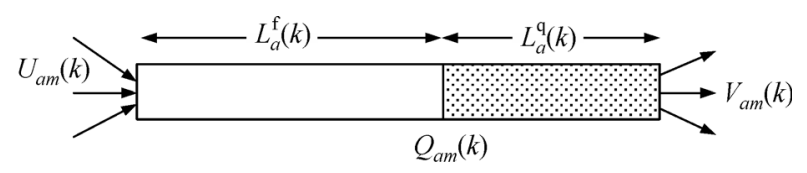

Fig. 1 The illustration for Link $a$ 
of vehicles of each type on each link and its queue area, given by

$$
X_{a m}(k)=U_{a m}(k)-V_{a m}(k),
$$

and

$$
X_{a m}^{\mathrm{q}}(k)=Q_{a m}(k)-V_{a m}(k) .
$$

The cumulative flows of each link can be disaggregated by the routes through the link, and the relationships between link-based flows and route-based flows are formulated as follows:

$$
\begin{aligned}
U_{a m}(k) & =\sum_{p} \delta_{a}^{\mathrm{p}} U_{a m p}(k), \\
Q_{a m}(k) & =\sum_{p} \delta_{a}^{\mathrm{p}} Q_{a m p}(k), \\
V_{a m}(k) & =\sum_{p} \delta_{a}^{\mathrm{p}} V_{a m p}(k), \\
Q_{a m}^{b}(k) & =\sum_{p} \delta_{a b}^{\mathrm{p}} Q_{a m p}(k), \\
V_{a m}^{\mathrm{b}}(k) & =\sum_{p} \delta_{a b}^{\mathrm{p}} V_{a m p}(k) .
\end{aligned}
$$

\subsection{The link model}

Since different types of vehicles have different characteristics such as width, length and height; one car is considered as passenger car unit (pcu) for convenience. In this paper, we suppose the queue density $J_{a}$ is given, then the queue length can be specified by

$$
L_{a}^{\mathrm{q}}(k)=\sum_{m} X_{a m}^{\mathrm{q}}(k) / J_{a}=\sum_{m}\left[Q_{a m}(k)-V_{a m}(k)\right] / J_{a} .
$$

By definition, we can obtain the length of the free flow area $L_{a}^{\mathrm{f}}(k)=L_{a}-L_{a}^{\mathrm{q}}(k)$. If $L_{a}^{\mathrm{q}}(k)=L_{a}$, queue spillback happens.

Since vehicles travel with free flow speed in the free flow area, for a special vehicle of type $m$, its entering time into Link $a$ must be $\delta\left(k+1-L_{a}^{\mathrm{f}}(k) / v_{a m}^{\mathrm{f}} \delta\right)$ if it leaves the free flow area into the queue area of Link $a$ at the end of time interval $k+1$. Therefore, the cumulative flows have the following relationship:

$$
Q_{a m}(k+1)=U_{a m}\left(k+1-L_{a}^{\mathrm{f}}(k) / v_{a m}^{\mathrm{f}} \delta\right) .
$$

$L_{a}^{\mathrm{f}}(k) / v_{a m}^{\mathrm{f}} \delta$ may not be an integer. If so, $k+1-L_{a}^{\mathrm{f}}(k) /$ $v_{a m}^{\mathrm{f}} \delta$ will also not be an integer. In this case, the linear interpolation method can be used to approximate the righthand side of Eq. (9).

\subsection{Node model}

As shown in Fig. 2, $A_{l}$ is the set of links leading to Node $l$, $B_{l}$ is the set of links leaving Node $l$. The node model depicts the flow propagation from Link $a \in A_{l}$ to Link $b \in B_{l}$.

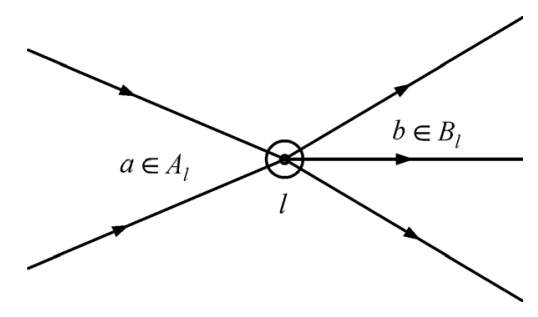

Fig. 2 An intersection sketch

For any link, the outflow is restricted by both the outflow capacity and the number of vehicles on the link. Those restrictions can be equivalently formulated as follows:

$$
\begin{aligned}
\sum_{m} V_{a m}(k+1) \leqslant & \min \left\{C_{a}^{\mathrm{out}}(k)+\sum_{m} V_{a m}(k),\right. \\
& \left.\sum_{m} U_{a m}\left(k+1-\frac{L_{a}}{v_{a m}^{\mathrm{f}} \delta}\right)\right\},
\end{aligned}
$$

where $C_{a}^{\text {out }}(k)$ is the outflow capacity of Link $a$ at time interval $k$. The first term on the right-hand side of Eq. (10) indicates that the total outflow of Link $a$ at time interval $k$ cannot exceed the outflow capacity, and the second term demonstrates that the cumulative outflow for vehicle of type $m$ by the end of time interval $k+1$ must enter the Link $a$ before time $\delta\left(k+1-L_{a} / v_{a m}^{\mathrm{f}} \delta\right)$.

Furthermore, the transmission flow from Link $a$ to Link $b$ is restricted by the receiving capacity of Link $b$. The receiving capacity depends on the inflow capacity and the accommodated vehicles' number of Link $b$. Therefore, the cumulative outflow from Link $a$ to Link $b$ should also satisfy the following condition:

$$
\begin{aligned}
\sum_{m} V_{a m}^{b}(k+1) \leqslant & p_{a}^{b}(k) \cdot \min \left\{C_{b}^{\mathrm{in}}(k), L_{b} J_{b}-\sum_{m} X_{b m}(k)\right\} \\
& +\sum_{m} V_{a m}^{b}(k),
\end{aligned}
$$

where $p_{a}^{b}(k)$ is the priority coefficient for transmission flow from Link $a$ to Link $b$ during time interval $k$. It is correlative with $C_{a}^{\text {out }}(k)$. If $C_{a}^{\text {out }}(k)=0$, we can adjust $p_{a}^{b}(k)$ $=0$. Anyway, we have $\sum_{a^{\prime}} p_{a^{\prime}}^{b}(k)=1 . C_{b}^{\text {in }}(k)$ is the inflow capacity of Link $b$ at time interval $k$, and $L_{b} J_{b}-\sum_{m} X_{b m}(k)$ 
is the maximum number of additional vehicles that can be contained in Link $b$ at the end of time interval $k$.

According to the length of queue, we consider the following two cases:

Case I: No queue (i.e., the queue length of Link $a$ equals zero)

In this case, vehicles travel with free flow speed to link exit. Limited to the travel time, the maximum cumulative number of vehicles allowed to leave Link $a$ equals the cumulative number of vehicles entering the link by time interval $k+1-L_{a} / v_{a m}^{\mathrm{f}} \delta$. Therefore, we have

$$
V_{\text {amp }}(k+1) \leqslant U_{\text {amp }}\left(k+1-L_{a} / v_{a m}^{\mathrm{f}} \delta\right) .
$$

Case II: Queuing (i.e., the queue length of Link $a$ is positive)

Since all vehicles obey FIFO rule in the queue area, there exists one certain time $t_{a k}$ such that $V_{a m p}(k+1)=$ $Q_{a m p}\left(t_{a k}\right)$. The vehicles leaving Link $a$ before the end of time interval $k+1$ have entered the queue area before time interval $t_{a k}$, but the vehicles entering the queue area after time $t_{a k}$ will continue to stay in Link $a$ by the end of time interval $k+1$. The following equation is used to determine $t_{a k}$ :

$$
\begin{aligned}
t_{a k}= & \max \left\{t: \sum_{m} Q_{a m}(t / \delta) \leqslant \min \left\{C_{a}^{\mathrm{out}}(k)+\sum_{m} V_{a m}(k),\right.\right. \\
& \left.\sum_{m} U_{a m}\left(k+1-\frac{L_{a}}{v_{a m}^{\mathrm{f}} \delta}\right)\right\}, \sum_{m} Q_{a m}^{b}(t / \delta) \\
& \leqslant p_{a}^{b}(k) \cdot \min \left\{C_{b}^{\mathrm{in}}(k), L_{b} J_{b}-\sum_{m} X_{b m}(k)\right\} \\
& \left.+\sum_{m} V_{a m}^{b}(k)\right\} .
\end{aligned}
$$

According to the conservation law, the cumulative inflow of Link $b$ belongs to Path $p$ equals to the corresponding cumulative outflow of its previous link on that path. For the special case, if Link $b$ is the first link on the path, the cumulative flow equals to the cumulative departure flow of the path. Therefore, we have

$$
\begin{aligned}
& U_{b m p}(k+1) \\
& = \begin{cases}\sum_{l=1}^{k+1} f_{m p}(l), & \text { if } b \text { is the first link on path } p, \\
V_{\text {amp }}(k+1), & \text { otherwise, }\end{cases}
\end{aligned}
$$

where Link $a$ is the previous link of Link $b$ on Path $p$.

\subsection{Simulation procedure}

From the above, the link model is used to calculate the cumulative inflow of the queue area, while the node model is applied to calculate the transmission flow from upstream links to downstream links and determine the cumulative inflow and outflow of each link. In summary, the detailed simulation procedure of the proposed model is outlined as follows:

Step 1: Initialize the cumulative flow at the start time to be zero, and set $k=0$;

Step 2: Calculate the link cumulative inflow $U_{a m}(k)$;

Step 2.1: Calculate $U_{\text {amp }}(k)$ with Eq. (14);

Step 2.2: Calculate $U_{a m}(k)$ with Eq. (3);

Step 3: Calculate the cumulative queue inflow $Q_{a m}(k+1)$;

Step 3.1: Calculate the length of queue part $L_{a}^{\mathrm{q}}(k)$ with Eq. (8), then the length of free flow part can be obtained;

Step 3.2: Calculate $Q_{a m}(k+1)$ with Eq. (9);

Step 4: Calculate the link cumulative outflow $V_{a m}(k+1)$;

Step 4.1: Calculate $V_{a m p}(k+1)$ with Eq. (12) or (13);

Step 4.2: Calculate $V_{a m}(k+1)$ with Eq. (5);

Step 5: If $k=M$ ( $M$ is the ending time interval), stop. Otherwise, $k=k+1$. Return to Step 2 .

\subsection{Link travel time estimation}

The cumulative link inflow and outflow can be obtained using the above flow propagation model, which can be used to estimate link travel times. The link travel time by the end of interval $k$ is defined as the horizontal distance between two cumulative flow curves by Lo and Szeto (2002a) and Huang and Lam (2002). In Fig. 3, we have the relationship $U_{a m}(k)=V_{a m}\left(k+\tau_{a m}(k)\right)$. Since the time is discretized, the vehicles entering the link at the end of time interval $k$ may leave the link at some time between $\left[k^{\prime} \delta,\left(k^{\prime}+1\right) \delta\right]$ or time interval $k^{\prime}+\mu_{m}\left(0<\mu_{m}<1\right)$, where $k^{\prime}>k$. Linear interpolation can be used to formulate

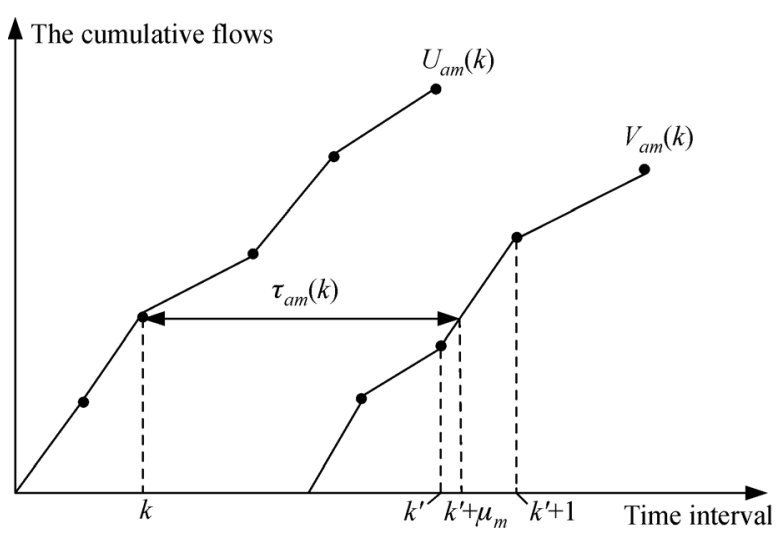

Fig. 3 Link cumulative inflow and outflow 
$U_{a m}(k), \quad U_{a m}(k)=\left(1-\mu_{m}\right) V_{a m}\left(k^{\prime}\right)+\mu_{m} V_{a m}\left(k^{\prime}+1\right)$. Using this linear interpolation, we can obtain $\mu_{m}$, given as follows

$$
\mu_{m}=\frac{U_{a m}(k)-V_{a m}\left(k^{\prime}\right)}{V_{a m}\left(k^{\prime}+1\right)-V_{a m}\left(k^{\prime}\right)} .
$$

By definition of $\mu_{m}$, we have $U_{a m}(k)=V_{a m}\left(k^{\prime}+\mu_{m}\right)$. According to the relationship $U_{a m}(k)=V_{a m}\left(k+\tau_{a m}(k)\right)$, we have $k^{\prime}+\mu_{m}=k+\tau_{a m}(k)$, which implies that the link travel time can be easily obtained by the following equation:

$$
\tau_{\text {am }}(k)=\left(k^{\prime}+\mu_{m}-k\right),
$$

where the unit of link travel time is time interval.

If function $V_{a m}(\cdot)$ is strictly monotonic increasing, then $\tau_{a m}(k)=V_{a m}^{-1}\left(U_{a m}(k)\right)-k$. However, there are two reasons that may cause the link cumulative inflow or cumulative outflow not to satisfy strictly monotone increasing: (1) A temporary inflow drops to zero (e.g., during light traffic), and (2) a temporary outflow capacity drops to zero (e.g., during a red phase, an incident or an accident). If one of the above situations happen, Eqs. (15) and (16) cannot be used to calculate the link travel time directly. In this case, a linear interpolation method developed by Nie (2003) can be adopted.

\section{Numerical simulation and analysis}

To illustrate the multi-class traffic flow propagation in link and in network and the difference between the models by Bliemer (2007) and the developed one, several numerical examples are applied to simulate the propagation with the proposed model.

3.1 Example 1: Comparison with the existing model (Bliemer, 2007)

To compare the difference between the improved model and the existing model, we give a very simple illustrative example. Suppose the traffic flow propagates only on one single link, and only one vehicle type. The vehicle inflow rate is set as $10 \mathrm{pcu} / \mathrm{s}$. Suppose the free-flow speed of vehicles is $180 \mathrm{~km} / \mathrm{h}$. The link length is $1 \mathrm{~km}$, and the freeflow travel time of vehicles is $20 \mathrm{~s}$. The queue density, the inflow capacity, and the outflow capacity of the link are $1000 \mathrm{pcu} / \mathrm{km}, 20 \mathrm{pcu} / \mathrm{s}$ and $20 \mathrm{pcu} / \mathrm{s}$ respectively. The length for each time interval $\delta=1 \mathrm{~s}$.

We consider two scenarios. The first scenario is described as follows: At a certain time slot $k$, the vehicles' number in the queue part is exactly $20 \mathrm{pcu}$. We can get different results by adopting the two models to implement the DNL. Figure 4 illustrates the resulting number of vehicles arriving at link exit, the amount of vehicles within the queue, the outflow capacity and the actual outflow of

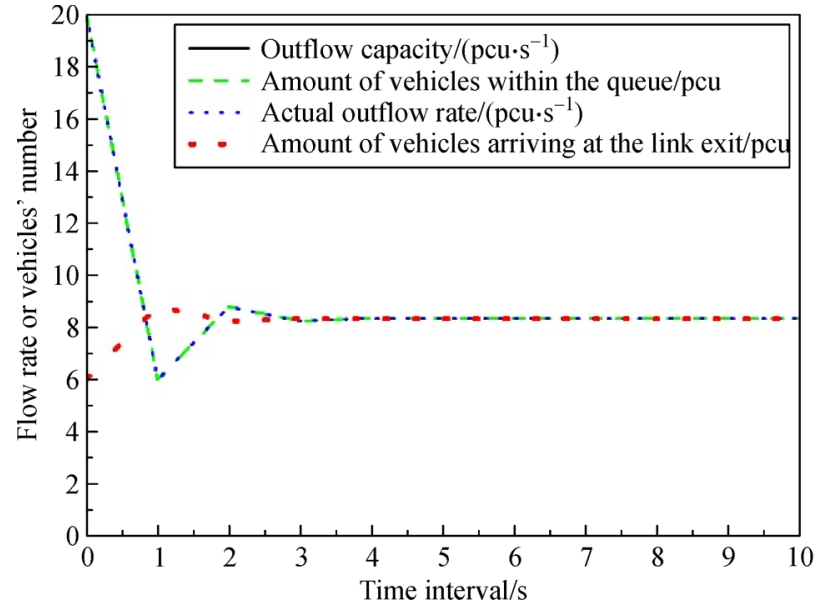

(a)

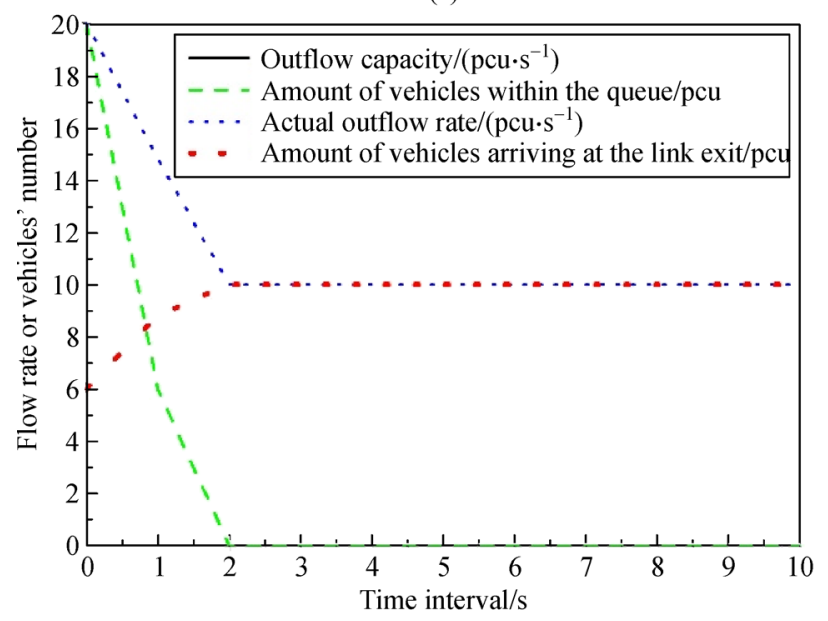

(b)

Fig. 4 Different results with two models (a) The results with Bliemer's model; (b) the results with the improved model

the link within 10 time slots after $k$.

By adopting Bliemer (2007)'s model, the results show that the outflow rate is always less than the vehicles' number in the queue part (Fig. 4(a)). The vehicles in the free part, which can leave at the same time interval, is not considered; these vehicles will form the queue at this time slot and will leave the link at the next time slot. However, in the improved model, we consider these vehicles; therefore, the outflow rate is greater than or equal to the result with Bliemer (2007)'s model (Fig. 4(b)). After $k+2$ $\mathrm{s}$, there is no queue part in the link.

In another scenario, we suppose at a certain time slot the queue length is near the link length, but there is still some available space with $5 \mathrm{pcu}$. The outflow capacity changes to 0 due to the red traffic light or other blocks. By adopting Bliemer (2007)'s model, the inflow rate should be 10 $\mathrm{pcu} / \mathrm{s}$. This is not suitable for the actual case. With the improved model, the inflow rate should be $5 \mathrm{pcu} / \mathrm{s}$ at this time slot. In fact, when the accommodated vehicles' number in the link is less than the inflow capacity, we 
should consider the smaller one.

3.2 Example 2: Interactions between multi-class traffic flows

This example considers the traffic flow propagation on a single link, and there are two vehicle types: trucks and cars. The inflow rates of cars in this example are given as follows:

$$
f_{1}(t)= \begin{cases}0.0185 t, & \text { if } t \leqslant 320 \mathrm{~s} \\ 0.05, & \text { if } 320 \mathrm{~s}<t \leqslant 800 \mathrm{~s}, \\ 0, & \text { if } t>800 \mathrm{~s}\end{cases}
$$

where the unit for the inflow is $\mathrm{pcu} / \mathrm{s}$. The inflow rates of trucks are one fifth that of the cars. Suppose the free-flow speeds of cars and trucks are 60 and $40 \mathrm{~km} / \mathrm{h}$, respectively. The link length is $2 \mathrm{~km}$, and the free-flow travel time of cars and trucks are 120 and $180 \mathrm{~s}$, respectively. The queue density, the inflow capacity, and the outflow capacity of the link are $360 \mathrm{pcu} / \mathrm{km}, 7200$ and $7200 \mathrm{pcu} / \mathrm{h}$, respectively. The simulation time horizon is $1000 \mathrm{~s}$, and the length for each time interval $\delta=1 \mathrm{~s}$.

The link travel time for cars and trucks in different time intervals is illustrated in Fig. 5. It can be observed that the link travel time for cars is always less than that of trucks. With increasing traffic flow in the link, the queue is formed gradually, and the difference between the two travel times becomes small. The longer the queue, the shorter the free flow area and the closer two travel times (Fig. 5). The length of the free flow area consistently varies with the difference between the two travel times.

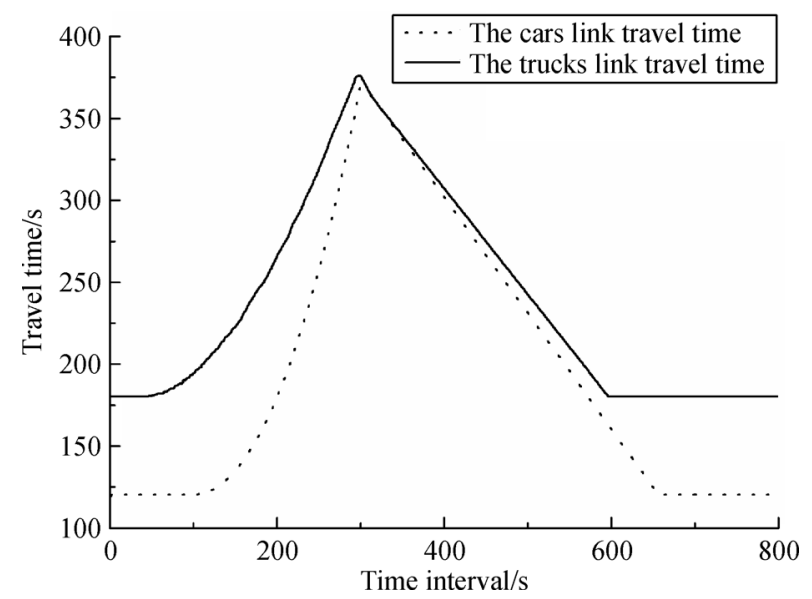

Fig. 5 Link travel times for cars and trucks

In Fig. 6, the link queue length is $0 \mathrm{~km}$ before times of $220 \mathrm{~s}$. This finding indicates that cars and trucks travel at the corresponding free traveling speed for the entire link before times of 100 and $40 \mathrm{~s}$, respectively. Therefore, before the time $40 \mathrm{~s}$, the difference between the two travel

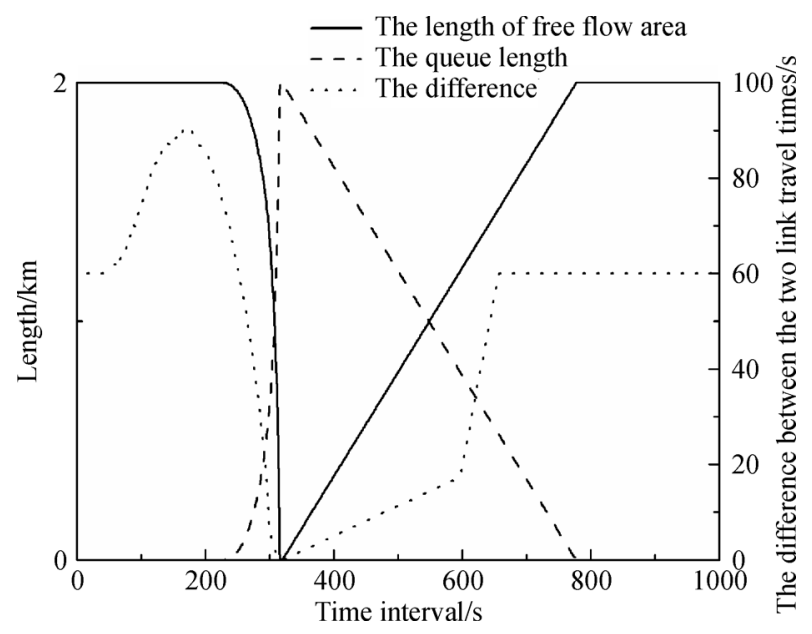

Fig. 6 Queue length and the difference between two travel times

times is $60 \mathrm{~s}$. However, the cars entering the link from a time of 40 to $100 \mathrm{~s}$ travel at the free traveling speed for the entire link, while the trucks entering the link at the same time period would encounter queuing. Therefore, the difference between the two travel times increases. With the traffic flow increasing, queuing influences the trucks more seriously than the cars.

The difference reaches a maximum at a time of $170 \mathrm{~s}$. However, with further increase in the traffic flow, the queue rapidly becomes longer, and the interaction between cars and trucks increases; which gradually reduces the difference of the two travel times. At a time of $320 \mathrm{~s}$, the queue length equals to the link length, and the two travel times are identical. After that time, the inflow of cars and trucks changes to 0.5 and $0.1 \mathrm{pcu} / \mathrm{s}$, respectively. The queue begins to dissipate, and the difference between the two travel times increases gradually. After the queue dissipates completely, the difference remains at $60 \mathrm{~s}$.

\subsection{Example 3: Traffic interactions across multiple links}

In general, the traffic flow propagation model based on physics queue can describe the interaction across multiple links, queue formation and dissipation, and queue spillback. The model developed in this paper can also describe the actual traffic flow characteristics.

A diverge network, shown in Fig. 7, is applied in Example 3. The length of Links 1 and 4 is $2 \mathrm{~km}$ and that of Links 2 and 3 is $1 \mathrm{~km}$. The queue density for Link 1 is 360 $\mathrm{pcu} / \mathrm{km}$, while others are $180 \mathrm{pcu} / \mathrm{km}$. The outflow capacity for Link 1 is set as $7200 \mathrm{pcu} / \mathrm{h}$, while others are set as $3600 \mathrm{pcu} / \mathrm{h}$. The inflow capacity equals the outflow capacity for each link.

The exit of Link 2 is controlled by traffic light signal. The traffic light signal's red light phase occurs from time 160 to $400 \mathrm{~s}$, and its green light phase occurs for all other times. Like Example 1, the two types of vehicles in the network are cars and trucks. The simulation time horizon is 


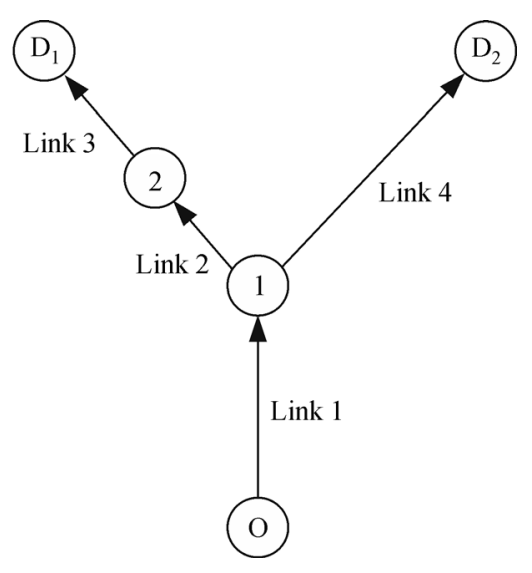

Fig. 7 Diverge network

also set as $1000 \mathrm{~s}$, and the length of each time interval is $\delta=1 \mathrm{~s}$. The demand of $\mathrm{OD}_{1}$ and $\mathrm{OD}_{2}$ is 1 and $0.6 \mathrm{pcu} / \mathrm{s}$ from time 0 to $500 \mathrm{~s}$, respectively.

Two simulation scenarios are considered. One is the propagation with the different mixed traffic flow ratios, and the other is the propagation with the different free traveling speed of trucks. Regardless of how the mixed traffic flow ratios change, the OD demands of traffic flow remains the same.

(1) The propagation with the different mixed traffic flow ratios.

The free traveling speed for cars and trucks is set as 60 and $40 \mathrm{~km} / \mathrm{h}$, respectively. Five different mixed traffic flow ratios of cars to trucks are considered. They are 4:1, 1:1, $1: 3$, only cars, and only trucks.

For each ratio the queue spillback occurs in Link 2, which leads to a queue forming in Link 1 as shown in Figs. 8 and 9. The larger the cars proportion in mixed traffic flow, the earlier queue spillback occurs in Link 2.

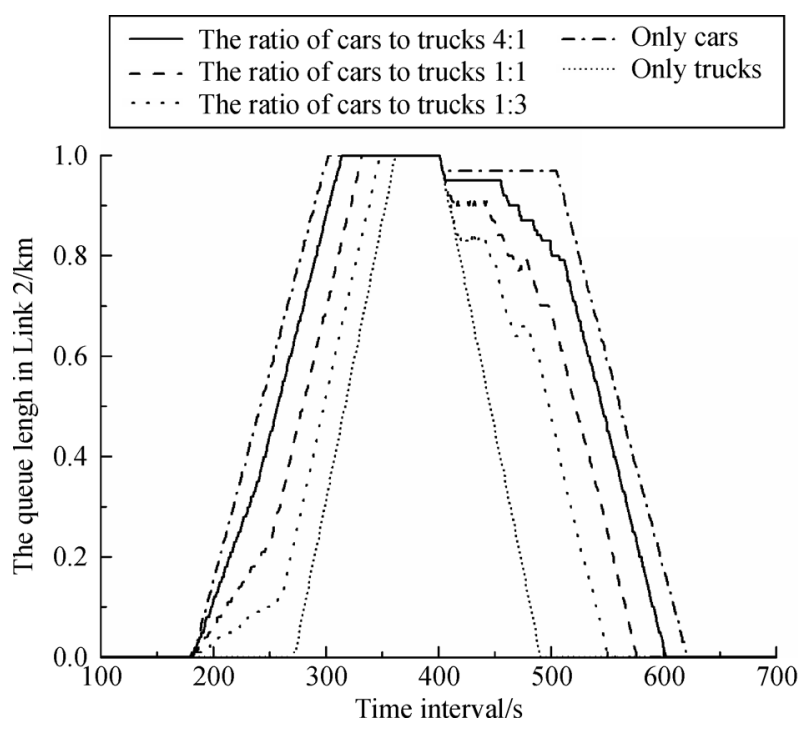

Fig. 8 Queue in Link 2 with different mixed traffic flow ratios

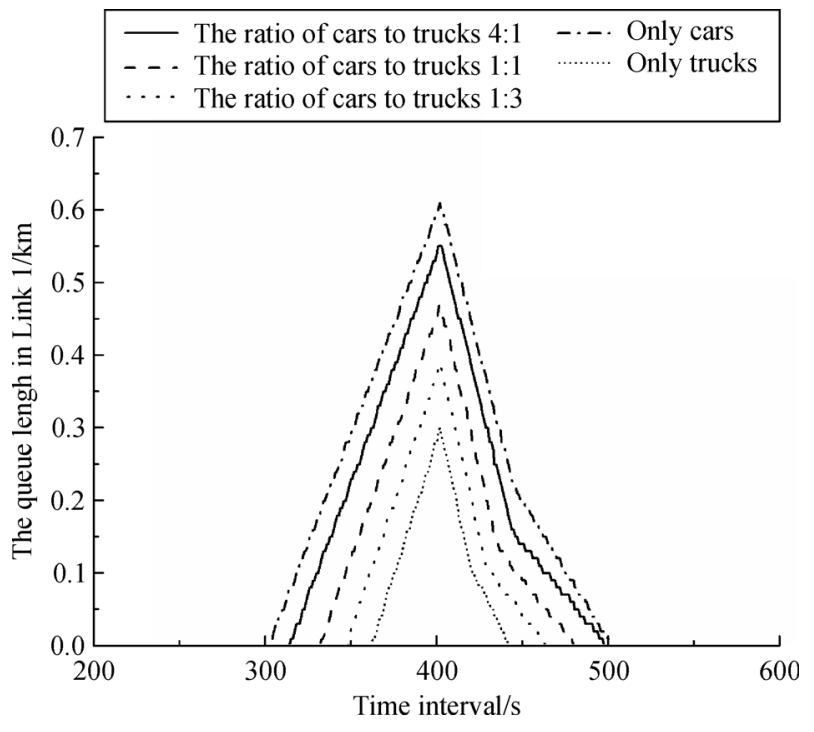

Fig. 9 Queue in Link 1 with different mixed traffic flow ratios

After spillback, the queue in Link 1 forms earlier and longer.

From a time of $400 \mathrm{~s}$, when the traffic light signal turns to green, the queue in Link 2 begins to dissipate. The larger the cars proportion in the mixed traffic flow, the later Link 1 reverts to free traveling state. The reason is that if the cars proportion in the mixed traffic flow is larger, more cars enter the queuing part early; and the queue in Link 2 forms earlier, and the queue length increases more rapidly. When the queue length equals the link length, queue spillback happens. With the larger car proportion, the queue in Link 1 forms earlier, the queue length is also longer, and the time for queue dissipation in Link 1 takes longer.

(2) The propagation with different free travel speed of trucks.

The free travel speed of cars is fixed as $v_{1 \mathrm{f}}=60 \mathrm{~km} / \mathrm{h}$. Consider 4 cases with different free traveling speed of trucks. The cases are 20,30, 40 and $50 \mathrm{~km} / \mathrm{h}$. The mixed traffic flow ratio of cars to trucks is set as 1:1.

The simulation results are shown in Figs. 10 and 11. It can be found that if the difference between the two free traveling speeds reduces, the queue spillback in Link 2 occurs earlier. Next, the queue length in Link 1 is longer, and later reverts to free travel state. The reason is that the queue forming initially in Link 2 is caused by car flow congestion. If the free traveling speed of trucks becomes larger, the trucks will enter the queue in Link 2 earlier and the queue in Link 2 increases more rapidly. It is shown as the dotted rising lines in Fig. 10.

Since the duration time of the red light is not long enough, when the free travel speed of trucks is $20 \mathrm{~km} / \mathrm{h}$ the trucks would not encounter queuing in Link 2 due to their slow speed. The vehicles in the queue only include cars. Meanwhile, the queue spillback in Link 2 would not happen. Correspondingly, the queue length in link 1 is zero. 


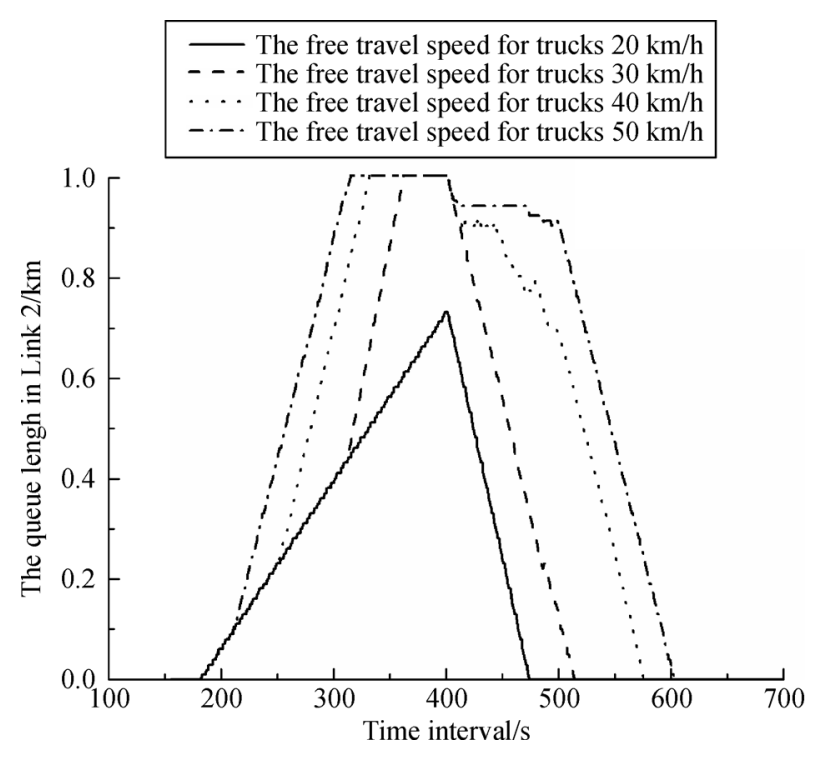

Fig. 10 Queue length in Link 2 with different free traffic speeds for trucks

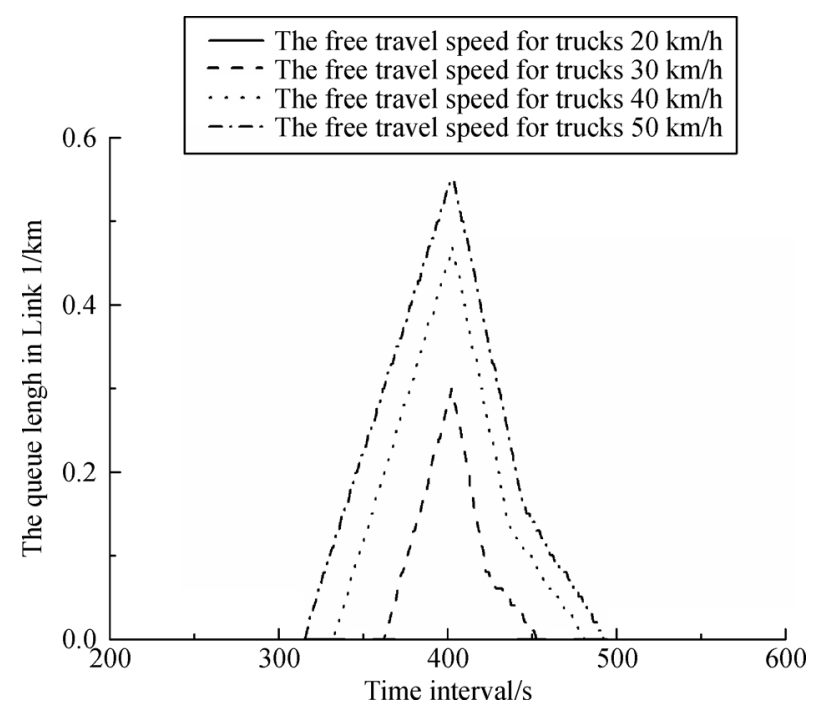

Fig. 11 Queue length in Link 1 with different free traffic speeds for trucks

\section{Conclusions}

In this paper, we present an improved multi-class dynamic network traffic flow propagation model with consideration of physical queues. Each whole link is divided into two parts (free flow part and queue part). Therefore, the model's computational efficiency is considerably higher than that of classic model based on the multiclass LWR theory, such as MCTM. The vehicles of the same class satisfy FIFO principle on the whole link, and the vehicles of the different classes also follow FIFO in the queue area but not in the free flow area. We improve the model by enhancing the sending and receiving functions of the model. The model is formulated in discrete time space directly for better solving. Several numerical examples are developed to illustrate that the developed model can more accurately capture the traffic flow propagations; such as interactions between multi-class traffic flows and the dynamic traffic interactions across multiple links. In the future studies, the proposed model can be adopted into DTA problems.

\section{Notations}

\begin{tabular}{|c|c|}
\hline$U_{a m}(k)$ & $\begin{array}{l}\text { The cumulative inflow of Link } a \text { for vehicle type } m \text { by the end of } \\
\text { time interval } k\end{array}$ \\
\hline$U_{\text {amp }}(k)$ & $\begin{array}{l}\text { e cumulative inflow of Link } a \text { belonging to Path } p \text { for vehicle } \\
m \text { by the end of time interval } k\end{array}$ \\
\hline$Q_{a m}(k)$ & of Link $a$ for vehicle type $m$ by the \\
\hline$Q_{a m p}(k)$ & $\begin{array}{l}\text { longing to Path } p \text { for } \\
k\end{array}$ \\
\hline$Q_{a m}^{b}(k)$ & vard Link $b$ for vehicle \\
\hline$V_{a m}(k)$ & $\begin{array}{l}\text { The cumulative outflow of Link } a \text { for vehicle type } m \text { by the end } \\
\text { of time interval } k\end{array}$ \\
\hline$V_{\text {amp }}(k)$ & g to Path $p$ for vehicle \\
\hline$V_{a m}^{b}$ & by the end of time interval $k$ \\
\hline$J_{a}$ & The queue density of Link $a /\left(\mathrm{pcu} \cdot \mathrm{km}^{-1}\right)$ \\
\hline$X_{a m}(k)$ & oer of vehicles in Link $a$ for ver \\
\hline$X_{a m}^{\mathrm{q}}(k)$ & $\begin{array}{l}\text { The number of vehicles in the queuing part of Link } a \text { for vehicle } \\
\text { type } m \text { at the end of time interval } k / \text { pcu }\end{array}$ \\
\hline$v_{a m}^{\mathrm{f}}$ & The free flow speed of Link $a$ for vehicles of type $m$ \\
\hline$\tau_{a m}(k)$ & $\begin{array}{l}1 \text { time for vehicles of type } m \text { entering Link } a \\
\text { terval } k\end{array}$ \\
\hline$\delta_{a}^{p}$ & $\delta_{a}^{p}=1$ if Link $a$ is on route $p$, and $\delta_{a}^{p}=0$ otherwise \\
\hline$\delta_{a b}^{p}$ & $\begin{array}{l}\delta_{a b}^{p}=1 \text { if Links } a \text { and } b \text { are on route } p \text {, and } a \text { is th } \\
\text { of } b \text {, and } \delta_{a b}^{p}=0 \text { otherwise }\end{array}$ \\
\hline
\end{tabular}

Acknowledgements This work was jointly supported by the National Natural Science Foundation of China (Grant Nos. 71571150 and 71361006), the Humanities and Social Science Foundation of The Ministry of Education (Grant No. 14YJA630026), and the Fundamental Research Funds for the Central Universities (Grant No. 26815WCX03). The authors are grateful to the reviewer for his/her constructive comments.

\section{References}

Bliemer M (2007). Dynamic queuing and spillback in analytical multiclass dynamic network loading model. Transportation Research Record: Journal of the Transportation Research Board, 2029: 14-21 Daganzo C F (1994). The cell transmission model: A dynamic 
representation of highway traffic consistent with the hydrodynamic theory. Transportation Research Part B: Methodological, 28(4): 269287

Daganzo C F (1995). The cell transmission model, Part II: Network traffic. Transportation Research Part B: Methodological, 29(2): 7993

Huang H J, Lam W H K (2002). Modeling and solving the dynamic user equilibrium route and departure time choice problem in network with queues. Transportation Research Part B: Methodological, 36(3): $253-273$

Huang H J, Lam W H K (2003). A multi-class dynamic user equilibrium model for queuing networks with advanced traveler information systems. Journal of Mathematical Modelling and Algorithms, 2(4): 349-377

Kuwahara M, Akamatsu T (2001). Dynamic user optimal assignment with physical queues for a many-to-many OD pattern. Transportation Research Part B: Methodological, 35(5): 461-479

Leclercq L, Laval J A (2007). A multiclass car-following rule based on the LWR model. In: Appert-Rolland C, Chevoir F, Gondret P, Lassarre S, Lebacque J P, Schreckenberg M, eds. Traffic and Granular Flow '07. Berlin: Springer, 735-753

Levin M W, Boyles S D (2016). A multi-class cell transmission model for shared human and autonomous vehicle roads. Transportation Research Part C, Emerging Technologies, 62: 103-116

Lighthill M J, Whitham G B (1955). On kinematic waves. II. A theory of traffic flow on long crowded roads. In: Proceedings of the Royal Society of London A: Mathematical, Physical and Engineering Sciences. The Royal Society, 229(1178): 317-345

Liu S, De Schutter B, Hellendoorn H (2013). Multi-class traffic flow and emission control for freeway networks. In: 16th International IEEE Conference on Intelligent Transportation Systems-(ITSC). IEEE, 2223-2228

Liu S, Hellendoorn H, De Schutter B (2017). Model predictive control for freeway networks based on multi-class traffic flow and emission models. IEEE Transactions on Intelligent Transportation Systems, 18 (2): $306-320$

Lo H, Ran B, Hongola B (1996). Multiclass dynamic traffic assignment model: Formulation and computational experiences. Transportation Research Record: Journal of the Transportation Research Board, 1537: 74-82
Lo H K, Szeto W Y (2002a). A cell-based variational inequality formulation of the dynamic user optimal assignment problem. Transportation Research Part B: Methodological, 36(5): 421-443

Lo H K, Szeto W Y (2002b). A cell-based dynamic traffic assignment model: Formulation and properties. Mathematical and Computer Modelling, 35(7-8): 849-865

Lo H K, Szeto W Y (2004). Modeling advanced traveler information services: Static versus dynamic paradigms. Transportation Research Part B: Methodological, 38(6): 495-515

Logghe S, Immers L H (2008). Multi-class kinematic wave theory of traffic flow. Transportation Research Part B: Methodological, 42(6): 523-541

Newell G F (1993). A simplified theory of kinematic waves in highway traffic, Part I: General theory; Part II: Queuing at freeway bottlenecks; Part III: Multi-destination flows. Transportation Research Part B: Methodological, 27(4): 281-314

Nie X (2003). The study of dynamic user-equilibrium traffic assignment. Dissertation for the Doctoral Degree. Davis: University of California, 242-255

Ran B, Boyce D (1996). Modeling Dynamic Transportation Network: An Intelligent Transportation System Oriented Approach. New York: Springer

Richards P I (1956). Shock waves on the highway. Operations Research, 4(1): 42-51

Szeto W Y, Jiang Y, Sumalee A (2011). A cell-based model for multiclass doubly stochastic dynamic traffic assignment. Computer-Aided Civil and Infrastructure Engineering, 26(8): 595-611

Tuerprasert K, Aswakul C (2010). Multiclass cell transmission model for heterogeneous mobility in general topology of road network. Journal of Intelligent Transport Systems, 14(2): 68-82

van Wageningen-Kessels F, Van Lint H, Hoogendoorn S, Vuik K (2010). Lagrangian formulation of multiclass kinematic wave model. Transportation Research Record: Journal of the Transportation Research Board, 2188: 29-36

van Wageningen-Kessels F (2016). Framework to assess multiclass continuum traffic flow models. Transportation Research Record: Journal of the Transportation Research Board, 2553: 150-160

Wong G C K, Wong S C (2002). A multi-class traffic flow model-An extension of LWR model with heterogeneous drivers. Transportation Research Part A, Policy and Practice, 36(9): 827-841 